\title{
On Certain Translation Invariant Properties of Interior Transmission Spectra and Their Doppler's Effect
}

\author{
Lung-Hui Chen \\ Department of Mathematics, National Chung Cheng University, 168 University Rd., Min-Hsiung, Chia-Yi County 621, Taiwan \\ Correspondence should be addressed to Lung-Hui Chen; mr.lunghuichen@gmail.com
}

Received 9 February 2017; Accepted 26 April 2017; Published 21 May 2017

Academic Editor: Hagen Neidhardt

Copyright (c) 2017 Lung-Hui Chen. This is an open access article distributed under the Creative Commons Attribution License, which permits unrestricted use, distribution, and reproduction in any medium, provided the original work is properly cited.

\begin{abstract}
We study the translation invariant properties of the eigenvalues of scattering transmission problem. We examine the functional derivative of the eigenvalue density function $\Delta(\hat{x})$ to the defining index of refraction $n(x)$. By the limit behaviors in frequency sphere, we prove some results on the inverse uniqueness of index of refraction. In physics, Doppler's effect connects the variation of the frequency/eigenvalue and the motion velocity/variation of position variable. In this paper, we proved the functional derivative $\partial_{r} \Delta(\widehat{x})=(1+\sqrt{n(r(\widehat{x}))}) / \pi$.
\end{abstract}

\section{Introduction}

In this paper, we investigate an inverse spectral problem in the following homogeneous interior transmission problem:

$$
\begin{array}{rlrl}
\Delta w+k^{2} n(x) w & =0, & & \text { in } D ; \\
\Delta v+k^{2} v & =0, & \text { in } D ; \\
w & =v, & \text { on } \partial D ; \\
\frac{\partial w}{\partial v} & =\frac{\partial v}{\partial v}, & & \text { on } \partial D,
\end{array}
$$

where $v$ is the unit outer normal; $D$ is a starlike domain in $\mathbb{R}^{3}$ containing the origin with boundary $\partial D ; n(x) \in \mathscr{C}^{2}\left(\mathbb{R}^{3}\right)$; $n(x)>0$, for $x \in D ; n(x)=1$, for $x \notin D ; n(0)=1$. Equation (1) is called the homogeneous interior transmission eigenvalue problem. We also assume the boundary is given and defined by

$$
R=R(\theta, \varphi) \in \mathscr{C}^{1}\left(\mathbb{S}^{2} ; \mathbb{R}^{+}\right),
$$

where $\mathbb{S}^{2}$ is the unit sphere with the spherical coordinate $\hat{x}:=(\theta, \varphi)$. Problem (1) occurs naturally when one considers the scattering of the plane waves hinging on certain inhomogeneity inside the domain $D$ that is defined by an index of refraction in many models. In the reduced scattering model (1), the inverse problem is to determine the index of refraction by the measurement of the scattered wave fields collected in the far fields. The inverse scattering problem plays a role in various disciplines of science and technology such as the applications in sonar and radar, geophysical sciences, astrophysics, medical imaging, remote sensing, and nondestructive testing in instrument manufacturing.

By an inhomogeneous interior transmission problem, we mean the following system:

$$
\begin{aligned}
\Delta w+k^{2} n(x) w & =0, \quad \text { in } D ; \\
\Delta v+k^{2} v & =0, \quad \text { in } D ; \\
w-v & =h_{l}^{(2)}(k r) Y_{l}^{m}(\widehat{x}), \quad \text { on } \partial D ; \\
\frac{\partial w}{\partial v}-\frac{\partial v}{\partial v} & =\frac{\partial}{\partial v}\left[h_{l}^{(2)}(k r) Y_{l}^{m}(\widehat{x})\right], \quad \text { on } \partial D,
\end{aligned}
$$

in which $h_{l}^{(2)}(k r)$ is the spherical Hankel function of order $l$ and $Y_{l}^{m}$ is the spherical harmonics of order $l, m$. We say $k \in \mathbb{C}$ is an interior transmission eigenvalue of (1) if there is a nontrivial pair of solution $w, v \in \mathscr{C}^{2}(D) \cap \mathscr{C}^{1}(\bar{D})$ to the boundary value problem (1). To ensure the uniqueness of 
the scattered solution outside $D$, we impose the Sommerfeld radiation condition; that is,

$$
\begin{gathered}
\lim _{r \rightarrow \infty} r\left(\frac{\partial w}{\partial r}-i k w\right)=0 \\
\lim _{r \rightarrow \infty} r\left(\frac{\partial v}{\partial r}-i k v\right)=0 .
\end{gathered}
$$

The interior transmission eigenvalues play a role in the inverse scattering theory both in numerical computation and in theoretical scattering theory. For the origin of interior transmission eigenvalue problem, we refer to Colton and Monk [1] and Kirsch [2]. For a theoretical study and historic literature, we refer to [3-6]. It is also another subject of research interest to study the existence or location of the eigenvalues [1, 4, 7-14]. It is expected to find a Weyl's type of asymptotics for the interior transmission eigenvalues. In this case, the distribution of transmission eigenvalues is directly related to certain spectral invariants of the scatterer. In this regard, we apply the methods from entire function theory [15-20] to study the distributional laws of the eigenvalues. We also refer to $[21,22]$ for the reconstruction of the interior transmission eigenvalues. For the nonsymmetrically stratified medium, there are not too many known results $[3,8,23]$. In this paper, we mainly follow the idea in $[9,24-26]$ to study the nonsymmetrical scatterers as a series of one-dimensional problems and consider the analytic continuation theorems of the Helmholtz equation. This paper aims to examine some spectral invariants associated to (1) and analyze the functional correspondence between the variation of the spectral density function and perturbation of the index of refraction.

\section{Preliminaries and Main Result}

Firstly, we expand the solution $(v, w)$ of $(1)$ in two series of spherical harmonics by Rellich's lemma. This is a Fourier type of expansion theory, and we refer to $[4$, p. 32, p. 227] and [14, p. 353].

$$
\begin{aligned}
& v(x ; k)=\sum_{l=0}^{\infty} \sum_{m=-l}^{m=l} a_{l, m} j_{l}(k r) Y_{l}^{m}(\widehat{x}) ; \\
& w(x ; k)=\frac{1}{r} \sum_{l=0}^{\infty} \sum_{m=-l}^{m=l} b_{l, m} y_{l}(r) Y_{l}^{m}(\widehat{x}),
\end{aligned}
$$

where

$$
\begin{aligned}
r & :=|x|, \\
R_{0} & \leq r<\infty, \\
\widehat{x} & =(\theta, \varphi) \in \mathbb{S}^{2},
\end{aligned}
$$

and $j_{l}$ is the spherical Bessel function of the first kind of order $l$. The summations converge uniformly and absolutely on compact subsets for sufficiently large $|x| \geq R_{0} \gg 0$. We refer the method to [4, p. 32, Lemma 2.11] which holds for a bounded perturbation.
Here, we note that the spherical harmonics

$$
\begin{array}{r}
Y_{l}^{m}(\theta, \varphi):=\sqrt{\frac{2 l+1}{4 \pi} \frac{(l-|m|) !}{(l+|m|) !}} P_{l}^{|m|}(\cos \theta) e^{i m \varphi}, \\
m=-l, \ldots, l ; l=0,1,2, \ldots,
\end{array}
$$

is a complete orthonormal system in $\mathscr{L}^{2}\left(\mathbb{S}^{2}\right)$, in which

$$
P_{n}^{m}(t):=\left(1-t^{2}\right)^{m / 2} \frac{d^{m} P_{n}(t)}{d t^{m}}, \quad m=0,1, \ldots, n,
$$

where the Legendre polynomials $P_{n}, n=0,1, \ldots$, form a complete orthogonal system in $L^{2}[-1,1]$. We refer this to [4, p. 25]. By the orthogonality of the spherical harmonics, the functions

$$
\begin{aligned}
v_{l, m}(x) & :=a_{l, m} j_{l}(k r) Y_{l}^{m}(\widehat{x}) ; \\
w_{l, m}(x) & :=\frac{b_{l, m} y_{l}(r)}{r} Y_{l}^{m}(\widehat{x})
\end{aligned}
$$

satisfy (1) independently for $|x| \geq R_{0}$. It is well-known that

$$
\begin{aligned}
\Delta_{\mathbb{R}^{3}}= & \frac{1}{r^{2}} \frac{\partial}{\partial r} r^{2} \frac{\partial}{\partial r}+\frac{1}{r^{2} \sin \varphi} \frac{\partial}{\partial \varphi} \sin \varphi \frac{\partial}{\partial \varphi} \\
& +\frac{1}{r^{2} \sin ^{2} \varphi} \frac{\partial^{2}}{\partial \theta^{2}},
\end{aligned}
$$

so we apply the Laplacian to the first equation of (1) for $|x| \gg$ $R_{0}$, where $n(x)=1$, and we observe that

$$
\begin{aligned}
\sum_{l=0}^{\infty} \sum_{m=-l}^{m=l} b_{l, m} \Delta_{\mathbb{R}^{3}} \frac{y_{l}(r)}{r} Y_{l}^{m}(\widehat{x}) & \\
= & \sum_{l=0}^{\infty} \sum_{m=-l}^{m=l} b_{l, m}\left(\frac{1}{r^{2}} \frac{\partial}{\partial r} r^{2} \frac{\partial}{\partial r} \frac{y_{l}(r)}{r}\right) Y_{l}^{m}(\widehat{x}) \\
& +\sum_{l=0}^{\infty} \sum_{m=-l}^{m=l} b_{l, m} \frac{y_{l}(r)}{r} \frac{1}{r^{2}} \Delta_{\mathbb{S}^{2}} Y_{l}^{m}(\widehat{x}) \\
= & -k^{2} n(x) \sum_{l=0}^{\infty} \sum_{m=-l}^{m=l} b_{l, m} \frac{y_{l}(r)}{r} Y_{l}^{m}(\widehat{x}) .
\end{aligned}
$$

Accordingly, the Fourier coefficients $y_{l}(r)$ trivially satisfy the following equation:

$$
y_{l}^{\prime \prime}+\left(k^{2} n(\widehat{x} r)-\frac{l(l+1)}{r^{2}}\right) y_{l}=0, \quad R_{0} \leq r<\infty .
$$

Most importantly, we extend the Fourier coefficients $y_{l}(r)$ along some fixed incident direction $\hat{x}$ into $|x| \leq R_{0}$ by considering the following scheme. Let us define

$$
\widehat{n}(r):=n(r \widehat{x}) .
$$

For the fixed $\hat{x} \in \mathbb{S}^{2}$, we consider the radially symmetric index of refraction in $\mathscr{C}^{2}\left(\mathbb{R}^{3}\right)$ by rotating $\widehat{n}(r)$ around the 
origin. Due to the radial-symmetry, the solution $y_{l}$ of (13) has an extension into $|x| \leq R_{0}$ depending on the incident direction $\hat{x}$ by solving the following ODE:

$$
\begin{gathered}
\widehat{y}_{l}^{\prime \prime}+\left(k^{2} \widehat{n}(r)-\frac{l(l+1)}{r^{2}}\right) \widehat{y}_{l}=0, \quad 0<r<R_{0} ; \\
\lim _{r \rightarrow 0}\left\{\frac{\widehat{y}_{l}(r)}{r}-j_{l}(k r)\right\}=0 .
\end{gathered}
$$

We denote the solution to $(15)$ as $\hat{y}_{l}(r ; k)$. We renormalize the initial condition as follows:

$$
\lim _{r \rightarrow 0}\left\{\frac{\widehat{y}_{l}(r)}{r}-j_{l}(k r)\right\}=0
$$

is independent of the incident direction $\widehat{x}$. We refer the initial condition to $[4,14]$.

Now we consider the following Liouville transformation for each fixed $\widehat{x} \in \mathbb{S}^{2}$ :

$$
\begin{aligned}
\widehat{z}_{l}^{i}\left(\widehat{\xi}^{i} ; k\right):= & {\left[n^{i}(r \widehat{x})\right]^{1 / 4} \widehat{y}_{l}(r ; k), } \\
& \text { where } \widehat{\xi}^{i}:=\int_{0}^{R(\widehat{x})}\left[n^{i}(\rho \widehat{x})\right]^{1 / 2} d \rho, i=1,2 .
\end{aligned}
$$

Let us define

$$
\widehat{B}^{i}:=\int_{0}^{R(\widehat{x})}\left[n^{i}(\rho \widehat{x})\right]^{1 / 2} d \rho, \quad i=1,2 .
$$

Then, we deduce from (15) and (17) that

$$
\left[\widehat{z}_{l}^{i}\right]^{\prime \prime}+\left[k^{2}-\widehat{p}^{i}\left(\widehat{\xi}^{i}\right)\right] \widehat{z}_{l}^{i}=0, \quad 0 \leq \widehat{\xi}^{i} \leq \widehat{B}^{i}, \quad i=1,2,
$$

in which

$$
\widehat{p}^{i}\left(\widehat{\xi}^{i}\right):=\frac{\left[\widehat{n}^{i}\right]^{\prime \prime}(r)}{4\left[\widehat{n}^{i}(r)\right]^{2}}-\frac{5}{16} \frac{\left\{\left[\widehat{n}^{i}\right]^{\prime}(r)\right\}^{2}}{\left[\widehat{n}^{i}(r)\right]^{3}}+\frac{l(l+1)}{r^{2} \widehat{n}^{i}(r)} .
$$

Here $\widehat{\xi}^{i}=\widehat{B}^{i}$ if and only if $r=R(\widehat{x})$ for the fixed $\widehat{x}$. Moreover,

$$
\begin{aligned}
\hat{q}^{i}\left(\widehat{\xi}^{i}\right):= & \frac{\left[\widehat{n}^{i}\right]^{\prime \prime}(r)}{4\left[\widehat{n}^{i}(r)\right]^{2}}-\frac{5}{16} \frac{\left\{\left[\widehat{n}^{i}\right]^{\prime}(r)\right\}^{2}}{\left[\widehat{n}^{i}(r)\right]^{3}}+\frac{l(l+1)}{r^{2} \widehat{n}^{i}(r)} \\
& -\frac{l(l+1)}{\left[\hat{\xi}^{i}\right]^{2}} .
\end{aligned}
$$

Thus, we deduce from (19), (20), and (21) that

$$
\left[\hat{z}_{l}^{i}\right]^{\prime \prime}+\left[k^{2}-\widehat{q}^{i}\left(\widehat{\xi}^{i}\right)-\frac{l(l+1)}{\left[\widehat{\xi}^{i}\right]^{2}}\right] \widehat{z}_{l}^{i}=0,
$$

For the simplicity of the notation, we drop the superscripts on the variables. When $l=0$, the asymptotic estimates for the solution $z_{0}(r)$ are classic and can be found in [27]

$$
\begin{aligned}
z_{0}(\xi ; k)= & \frac{\sin k \xi}{k}-\frac{\cos k \xi}{2 k^{2}} Q(\xi) \\
& +\frac{\sin k \xi}{4 k^{3}}\left[p(\xi)+p(0)-\frac{1}{2} Q^{2}(\xi)\right] \\
& +O\left(\frac{\exp [|\Im k| \xi]}{k^{4}}\right),
\end{aligned}
$$

where $Q(\xi):=\int_{0}^{\xi} p(s) d s$, and the error term can be improved [27, p. 17] by

$$
\begin{aligned}
& \frac{\cos k \xi}{8 k^{4}}\left\{p^{\prime}(\xi)-p^{\prime}(0)-[p(\xi)+p(0)] Q(\xi)\right. \\
& \left.\quad-\int_{0}^{\xi} p^{2}(s) d s+\frac{1}{6} Q^{3}(\xi)\right\}+o\left(\frac{\exp |\Im k| \xi}{|k|^{4}}\right) .
\end{aligned}
$$

Similarly,

$$
\begin{aligned}
z_{0}^{\prime}(\xi ; k)= & \cos k \xi+\frac{\sin k \xi}{2 k} Q(\xi) \\
& +\frac{\cos k \xi}{4 k^{2}}\left[p(\xi)-p(0)-\frac{1}{2} Q^{2}(\xi)\right] \\
& +O\left(\frac{\exp [|\Im k| \xi]}{k^{3}}\right)
\end{aligned}
$$

in which the boundary behavior of $p(\xi)$ plays a role in determining the inverse spectral uniqueness on the scatterer, and thus $\mathscr{C}^{2}$-assumption on the index of refraction is convenient.

For $l \geq-1 / 2$, there are much more generalized results from $[28,29]$. In particular, the solution of $(15)$ is an entire function of order one and of finite type [4, 9, 10, 14, 27-30] that has a Sturm-Liouville type of spectral analysis.

Now we fulfill the boundary conditions in (1). The nontrivial solutions (10) of the homogeneous system are required to satisfy the boundary condition along the fixed $\hat{x}$ as follows: for $-l \leq m \leq l, l=0,1,2, \ldots$,

$$
\begin{array}{r}
\left.\widehat{a}_{l, m} j_{l}(k r)\right|_{r=R}-\left.\widehat{b}_{l, m} \frac{\widehat{y}_{l}(r)}{r}\right|_{r=R}=0 ; \\
\left.\widehat{a}_{l, m}\left[j_{l}(k r)\right]^{\prime}\right|_{r=R}-\left.\widehat{b}_{l, m}\left[\frac{\widehat{y}_{l}(r)}{r}\right]^{\prime}\right|_{r=R}=0,
\end{array}
$$

in which we note that $R=R(\widehat{x})$. The eigenvalues are possibly the intersection points of two complex-valued functions, and the constants $\widehat{a}_{l, m}$ and $\widehat{b}_{l, m}$ depend on $\widehat{x}$ as well. Let us define

$$
\widehat{D}_{l}(k):=\operatorname{det}\left(\begin{array}{cc}
\left.j_{l}(k r)\right|_{r=R} & -\left.\frac{\widehat{y}_{l}(r)}{r}\right|_{r=R} \\
\left.\left\{j_{l}(k r)\right\}^{\prime}\right|_{r=R}-\left.\left\{\frac{\widehat{y}_{l}(r)}{r}\right\}^{\prime}\right|_{r=R}
\end{array}\right),
$$


in which $\widehat{D}_{l}(k)$ is independent of index $m$. The existence of the nontrivial $\left(\widehat{a}_{l, m}, \widehat{b}_{l, m}\right)$ of $(26)$ is equivalent to finding the zeros of

$$
\widehat{D}_{l}(k)=0 .
$$

The following fundamental lemma connects the zeros of $\widehat{D}_{l}(k)=0$ and the eigenvalues of (1).

Lemma 1. Let $k$ be an eigenvalue of (1) if and only if it satisfies (28) for all $l$ and some $\hat{x} \in \mathbb{S}^{2}$.

Proof. Let $v(x ; k)$ and $w(x ; k)$ be a nontrivial pair of solutions of (1). Then, (6) holds and uniquely defines the coefficients $\widehat{y}_{l}(r ; k)$ and all $l \geq 0$ for $r \geq R_{0}$ and extends into $|x| \leq R_{0}$ by solving (15) with some fixed $\widehat{x}$. The solution,

$$
w_{l, m}(x):=\frac{b_{l, m} y_{l}(r)}{r} Y_{l}^{m}(\widehat{x}), \quad \forall l \geq 0,
$$

each satisfies the Helmholtz equation independently and meets with $v_{l, m}(x)$ in $|x| \gg R_{0}$. By the analytic continuation of the Helmholtz equation [1], they extend to meet on $\partial D$. That is, (28) is satisfied at $\hat{x} \in \mathbb{S}^{2}$. Hence, (28) holds on $\hat{x}$ for all $l \geq 0$.

For the sufficient condition, if (28) is valid on the boundary for some $\tilde{x}$, then $\tilde{y}_{l}(r)$ define a Fourier coefficient by ODE (15) by some eigenvalue $k_{0}$, which defines a solution of the Helmholtz equation

$$
w_{l, m}\left(x ; k_{0}\right):=\frac{b_{l, m} \widetilde{y}_{l}\left(r ; k_{0}\right)}{r} Y_{l}^{m}(\widehat{x}), \quad \forall l \geq 0,
$$

for $|x| \geq R_{0}$. By the analytic extension of the Helmholtz equation, $w_{l, m}\left(x ; k_{0}\right)$ is defined outside $D$ and meets $v_{l, m}\left(x ; k_{0}\right)$ on $\partial D$. That is, (28) holds for all other $\hat{x} \in \mathbb{S}^{2}$ with $k_{0}$. Hence,

$$
\begin{aligned}
\left.\tilde{a}_{l, m} j_{l}\left(k_{0} r\right)\right|_{r=R} & =\left.\widetilde{b}_{l, m} \frac{\hat{y}_{l}\left(r ; k_{0}\right)}{r}\right|_{r=R} ; \\
\left.\tilde{a}_{l, m}\left[j_{l}\left(k_{0} r\right)\right]^{\prime}\right|_{r=R} & =\left.\widetilde{b}_{l, m}\left[\frac{\hat{y}_{l}\left(r ; k_{0}\right)}{r}\right]^{\prime}\right|_{r=R}, \quad \hat{x} \neq \tilde{x},
\end{aligned}
$$

which we consider as an initial condition for that Bessel function $j_{l}\left(k_{0} r\right)$, are known functions. Combining the initial condition with

$$
\widehat{y}_{l}^{\prime \prime}+\left(k^{2} \widehat{n}(r)-\frac{l(l+1)}{r^{2}}\right) \widehat{y}_{l}=0,
$$

we find that the solution $w\left(x ; k_{0}\right)$ defines an eigenfunction to (1). We refer more detailed construction to [11].

For $l=0, \widehat{D}_{l}(k)$ is easier to compute [24, (2.10)]:

$$
\widehat{D}_{0}(k)=\frac{\sin \{k R\}}{k} \hat{y}_{0}^{\prime}(R ; k)-\cos \{k R\} \widehat{y}_{0}(R ; k),
$$

in which all four elements have zeros distributed asymptotically like sine and cosine functions.
Moreover, $\widehat{D}_{0}(k)$ has the following Hadamard representation:

$$
\widehat{D}_{0}(k)=\widehat{\gamma} k^{\widehat{d}} \prod_{j}\left(1-\frac{k}{\widehat{k}_{j}}\right),
$$

where $\widehat{\gamma}$ is a constant $[24,(2.14)]$. From $[9,10]$, the zeros spread along the real axis by Cartwright theory $[19$, Ch. 6$]$ and $[18,20]$. In general $l \geq-1 / 2$, we state the following lemma.

Lemma 2. For the fixed $\hat{x} \in \mathbb{S}^{2}$, the determinant $\widehat{D}_{l}(k)$ has only finitely many different zeros to the ones of $k j_{l}^{\prime}(k) \hat{y}_{l}(R ; k)$ or $j_{l}(k R) y_{l}^{\prime}(R ; k)$ in each parallel adjacent strips to the imaginary axis.

Proof. We infer from (27) that

$$
\begin{aligned}
& \widehat{D}_{l}(k)=-\frac{j_{l}(k R) \hat{y}_{l}^{\prime}(R ; k)}{R}+\frac{j_{l}(k R) \widehat{y}_{l}(R ; k)}{R^{2}} \\
& +\frac{k j_{l}^{\prime}(k R) \widehat{y}_{l}(R ; k)}{R} ; \\
& \widehat{D}_{l}(k)=\frac{k j_{l}^{\prime}(k R) \widehat{y}_{l}(R ; k)}{R}\left\{1-\frac{1}{k} \frac{j_{l}(k R)}{j_{l}^{\prime}(k R)} \frac{\hat{y}_{l}^{\prime}(R ; k)}{\widehat{y}_{l}(R ; k)}\right. \\
& \left.+\frac{1}{k R} \frac{j_{l}(k R)}{j_{l}^{\prime}(k R)}\right\},
\end{aligned}
$$

in which we recall from [9] and [10, Lemma 2.5] that $j_{l}(k R) / j_{l}^{\prime}(k R)=O(1)$ and $\hat{y}_{l}^{\prime}(R ; k) / \widehat{y}_{l}(R ; k)=O(k)$ outside the zeros in the denominators. Without loss of generality, we drop the lower order term $(1 / k)\left(j_{l}(k R) / j_{l}^{\prime}(k R)\right)$ in the bracket in (36) and the term $1-(1 / k)\left(j_{l}(k R) / j_{l}^{\prime}(k R)\right)\left(\hat{y}_{l}^{\prime}(R ; k) / \widehat{y}_{l}(R\right.$; $k)$ ) is bounded and bounded away from zero near the zeros of $j_{l}^{\prime}(k R)$ and $\widehat{y}_{l}(R ; k)$. The asymptotic expansion of $\widehat{y}_{l}(R ; k)$ for large $k$ is given in (23) and in [28,29]. In particular, $\widehat{D}_{l}(k)$ is bounded near the real axis and then in the Cartwright class [19, p. 251].

More importantly, we drop the lower order term in (35) and then consider Rouchés Theorem for the identity

$$
\left|\widehat{D}_{l}(k ; R)-k j_{l}^{\prime}(k R) \frac{\widehat{y}_{l}(R ; k)}{R}\right|=\left|j_{l}(k R) \frac{y_{l}^{\prime}(R ; k)}{R}\right| \text {. }
$$

The inequality

$$
\left|j_{l}(k R) \frac{y_{l}^{\prime}(R ; k)}{R}\right|<\left|k j_{l}^{\prime}(k R) \frac{\widehat{y}_{l}(R ; k)}{R}\right|
$$

holds if and only if

$$
\left|\frac{\hat{y}_{l}^{\prime}(R ; k)}{\widehat{y}_{l}(R ; k)}\right|<\left|k \frac{j_{l}^{\prime}(k R)}{j_{l}(k R)}\right| .
$$

We recall that

$$
\begin{aligned}
\frac{j_{l}(k R)}{j_{l}^{\prime}(k R)} & =\frac{\sin k}{\cos k}\left[1+O\left(\frac{1}{k}\right)\right] ; \\
\frac{\hat{y}_{l}^{\prime}(R ; k)}{\widehat{y}_{l}(R ; k)} & =\frac{k \cos k \widehat{B}}{\sin k \widehat{B}}\left[1+O\left(\frac{1}{k}\right)\right],
\end{aligned}
$$


outside their poles, where

$$
\widehat{B}:=\int_{0}^{R(\widehat{x})} \sqrt{n(\widehat{x} \rho)} d \rho .
$$

Hence, (39) holds if and only if

$$
|\cot k \widehat{B}|<|\cot k R|, \quad \text { for large } k \text {, }
$$

which are two asymptotically periodic meromorphic functions along the real axis. Referring the graph of $|\cot k|$ to [31], there are vertical lines in $\mathbb{C}$ passing through the real axis at which $|\cot k|=0$ and then strictly increases to 1 or at which $|\cot k|=\infty$ and strictly decreases to 1 , as $|\mathfrak{s} k|$ goes to infinity. For large $k$, there are infinitely many vertical lines of this property on which the following inequality holds:

$$
\left|\widehat{D}_{l}(k ; R)-k j_{l}^{\prime}(k R) \frac{\widehat{y}_{l}(R ; k)}{R}\right|<\left|k j_{l}^{\prime}(k R) \frac{\widehat{y}_{l}(R ; k)}{R}\right| \text {. }
$$

Similarly, we may repeat the argument from (37) to deduce that there are infinitely many vertical lines in $\mathbb{C}$ on which the following equality holds:

$$
\left|\widehat{D}_{l}(k ; R)-j_{l}(k R) \frac{y_{l}^{\prime}(R ; k)}{R}\right|<\left|j_{l}(k R) \frac{y_{l}^{\prime}(R ; k)}{R}\right| .
$$

Therefore, we apply Rouché's theorem to (43) and (44) on the boundary of a family of suitable vertical strips along the real axis and conclude that $\widehat{D}_{l}(k)$ have only finite many of different zeros to the ones of $k j_{l}^{\prime}(k) \widehat{y}_{l}(R ; k)$ or $j_{l}(k R) y_{l}^{\prime}(R ; k)$ in the strips.

One step further, we can describe the zero distribution of $\widehat{D}_{l}(k)$ more precisely by applying Wilder's theorem $[32,33]$ to the term $k j_{l}^{\prime}(k) \widehat{y}_{l}(R ; k)$ or $j_{l}(k R) y_{l}^{\prime}(R ; k)$ in (35) and obtain the following density distribution theorem for (33): let $R_{11}$, $R_{12}$, and $R_{13}$ be three adjacent strips containing all but a finite exceptions of the zeros along the positive real axis, and let $N\left(R_{1 i}(\alpha, s, K)\right), i=1,2,3$, be the zero counting function in the strip starting with $\Im k=\alpha$, of length $s$ and of width $K$. Then there exist some $K>0$ and large $\alpha$ such that

$$
\begin{aligned}
\widehat{B} & :=\int_{0}^{R(\widehat{x})} \sqrt{n(\widehat{x} \rho)} d \rho ; \\
N\left(R_{11}(\alpha, s, K)\right) & \sim \begin{cases}s \frac{\widehat{B}}{\pi}, & R(\widehat{x}) \geq \widehat{B} ; \\
s \frac{R(\widehat{x})}{\pi}, & R(\widehat{x})<\widehat{B} ;\end{cases} \\
N\left(R_{12}(\alpha, s, K)\right) & \sim s \frac{|R(\widehat{x})-\widehat{B}|}{\pi} ; \\
N\left(R_{13}(\alpha, s, K)\right) & \sim \begin{cases}s \frac{\widehat{B}}{\pi}, & R(\widehat{x}) \geq \widehat{B} ; \\
s \frac{R(\widehat{x})}{\pi}, & R(\widehat{x})<\widehat{B} .\end{cases}
\end{aligned}
$$

A similar argument holds for $j_{l}(k R) y_{l}^{\prime}(R ; k)$ that yields identical asymptotics to (46), (47), and (48). We refer the details to [10, Theorem 4.2].
From the point of view of the inhomogeneous system (3),

$$
\begin{gathered}
\left.\widehat{a}_{l, m} j_{l}(k r)\right|_{r=R}-\left.\widehat{b}_{l, m} \frac{\widehat{y}_{l}(r)}{r}\right|_{r=R} \\
=\left.\left[h_{l}^{(2)}(k r) Y_{l}^{m}(\widehat{x})\right]\right|_{r=R} ; \\
\left.\widehat{a}_{l, m} \frac{d}{d r} j_{l}(k r)\right|_{r=R}-\left.\widehat{b}_{l, m} \frac{d}{d r} \frac{\widehat{y}_{l}(r)}{r}\right|_{r=R} \\
=\left.\frac{d}{d r}\left[h_{l}^{(2)}(k r) Y_{l}^{m}(\widehat{x})\right]\right|_{r=R},
\end{gathered}
$$

to which we can solve for $\widehat{a}_{l, m}, \widehat{b}_{l, m}$ by Cramer's rule:

$$
\begin{aligned}
& \widehat{a}_{l, m}(k)=\frac{\operatorname{det}\left(\begin{array}{cc}
\widehat{y}_{l}(R) & {\left.\left[h_{l}^{(2)}(k r) Y_{l}^{m}(\widehat{x})\right]\right|_{r=R}} \\
\left.\left\{\widehat{y}_{l}(r) / r\right\}^{\prime}\right|_{r=R} & \left.(d / d r)\left[h_{l}^{(2)}(k r) Y_{l}^{m}(\widehat{x})\right]\right|_{r=R}
\end{array}\right)}{\widehat{D}_{l}(k)} ; \\
& \widehat{b}_{l, m}(k)=\frac{\operatorname{det}\left(\begin{array}{cc}
{\left.\left[h_{l}^{(2)}(k r) Y_{l}^{m}(\widehat{x})\right]\right|_{r=R}} & -j_{l}(k R) \\
\left.(d / d r)\left[h_{l}^{(2)}(k r) Y_{l}^{m}(\hat{x})\right]\right|_{r=R} & -\left.j_{l}^{\prime}(k r)\right|_{r=R}
\end{array}\right)}{\widehat{D}_{l}(k)},
\end{aligned}
$$

where $\widehat{a}_{l, m}(k), \widehat{b}_{l, m}(k)$ are understood as two meromorphic functions. An interior transmission eigenvalue means that the inhomogeneous system (3) can not be solved explicitly in $\mathscr{C}^{2}(D) \cap \mathscr{C}^{1}(\bar{D})$ whenever $\widehat{D}_{l}(k)$ has a zero in the denominator. We observe that $(w(r ; k), v(r ; k))$ is not a solution in the classic sense if there is any $l$ and any $\widehat{x}$ such that $\widehat{D}_{l}(k)$ has a zero. Whenever that happens, we have an interior transmission eigenvalue.

Definition 3. We define $\mathscr{Z}(\widehat{x})$ to be the union of the zero set of entire function $\widehat{D}_{0}(k)$ at $\widehat{x}$ and $-\widehat{x}$.

We need the following vocabulary to describe the asymptotic behavior of the eigenvalues.

Definition 4. Let $f(z)$ be an integral function of order $\rho$, and $N(f, \alpha, \beta, r)$ denote the number of the zeros of $f(z)$ inside the angle $[\alpha, \beta]$ and $|z| \leq r$; we define the zero density function

$$
\begin{aligned}
\Delta_{f}(\alpha, \beta) & :=\lim _{r \rightarrow \infty} \frac{N(f, \alpha, \beta, r)}{r^{\rho}}, \\
\Delta_{f}(\beta) & :=\Delta_{f}\left(\alpha_{0}, \beta\right),
\end{aligned}
$$

with some fixed $\alpha_{0} \notin E$ such that $E$ is at most a countable set [18-20].

Following the results in $[9-11,26]$, we have the density asymptotics:

$$
\begin{aligned}
\Delta_{\widehat{D}_{0}}(-\epsilon, \epsilon) & =\Delta_{\widehat{D}_{0}}(\pi-\epsilon, \pi+\epsilon) \\
& =\frac{R(\widehat{x})+\int_{0}^{R(\widehat{x})} \sqrt{n(\widehat{x} \rho)} d \rho}{\pi} ; \\
\Delta_{\widehat{D}_{0}}(\epsilon, \pi-\epsilon) & =\Delta_{\widehat{D}_{0}}(\pi+\epsilon, 2 \pi-\epsilon)=0 .
\end{aligned}
$$

For a simpler notation, we use

$$
\Delta(\widehat{x}):=\Delta_{\mathscr{L}(\widehat{x})}(-\epsilon, \epsilon)
$$

to denote the density of zero set of $\mathscr{Z}(\widehat{x})$. 
One can show the following.

Lemma 5. Let us denote

$$
\widehat{\mathscr{B}}:=\int_{-R(-\widehat{x})}^{R(\widehat{x})} \sqrt{n(\widehat{x} \rho)} d \rho,
$$

and the following identity holds:

$$
\Delta(\widehat{x})=\frac{R(\widehat{x})+R(-\widehat{x})+\widehat{\mathscr{B}}}{\pi} .
$$

Accordingly, the density function is invariant to the rigid translations of $D$.

Proof. We observe that

$$
\int_{0}^{R(\widehat{x})} \sqrt{n(\widehat{x} \rho)} d \rho+\int_{0}^{R(-\widehat{x})} \sqrt{n(-\widehat{x} \rho)} d \rho=\widehat{\mathscr{B}} .
$$

By applying (52) in (54) to $\hat{x}$ and $-\hat{x}$, respectively, (56) is proven. The invariant property comes from the translation transformation along $r$ of integral $\widehat{\mathscr{B}}$. The invariant property on rotation is trivial.

We state the inverse spectral uniqueness in this paper.

Theorem 6. Let $n^{1}, n^{2}$ be two unknown indices of refraction parameterized in (1) in domains $D^{1}$ and $D^{2}$ with eigenvalue sets $\mathscr{Z}^{1}(\widehat{x})$ and $\mathscr{Z}^{2}(\widehat{x})$, respectively, as defined in Definition 3. $D^{1}$ and $D^{2}$ are assumed to be identical up to a translation in $(r, \widehat{x}) \in \mathbb{R}^{+} \times \mathbb{S}^{2}$. If $\mathscr{Z}^{1}(\widehat{x})=\mathscr{Z}^{2}(\widehat{x})$ for infinitely many pairs of $(\widehat{x},-\widehat{x}) \in \mathbb{S}^{2} \times \mathbb{S}^{2}$, then $n^{1} \equiv n^{2}$ up to a translation in $(r, \widehat{x}) \in$ $\mathbb{R}^{+} \times \mathbb{S}^{2}$.

This is one local assumption implying a global uniqueness on the index of refraction. Previously [9, 10, 26], we have shown that if the interior transmission eigenvalues are given identical for all possible incident angles with $n^{1}(0)=n^{2}(0)=$ 1 , then we can prove the inverse uniqueness. Here we are given a potpourri of spectral data.

\section{Analysis along an Angle}

The following results hold up to a rigid translation in $\mathbb{R}^{+} \times \mathbb{S}^{2}$.

Lemma 7. Let $\Delta^{i}(\hat{x})$ be the density function of $n^{i}$ defined as in (54), $i=1,2$. Up to a translation in $(r, \widehat{x})$, there exists at least one accumulation point of pair of common incident directions $\left\{\widehat{x}_{0},-\widehat{x}_{0}\right\}$ and $\left\{\widehat{x}_{0}^{\prime},-\widehat{x}_{0}^{\prime}\right\} \in \mathbb{S}^{2}$ from $n^{1}$ and $n^{2}$, respectively, such that $\Delta^{1}(\hat{x})=\Delta^{2}\left(\widehat{x}^{\prime}\right)$ holds for $\widehat{x}$ near $\widehat{x}_{0}$ and $\widehat{x}^{\prime}$ near $\widehat{x}_{0}^{\prime}$, respectively, in $\mathbb{S}^{2}$.

Proof. $\mathbb{S}^{2}$ is compact. By applying the assumption in Theorem 6 , there are the accumulation points $\widehat{x}_{0}$ and $\hat{x}_{0}^{\prime}$ in $\mathbb{S}^{2}$, respectively, such that $\Delta^{1}(\hat{x})=\Delta^{2}\left(\widehat{x}^{\prime}\right)$ holds near $\widehat{x}_{0}$ and $\widehat{x}_{0}^{\prime}$, respectively, in $\mathbb{S}^{2}$. This proves the lemma.
Lemma 8. Let $R^{i}(\widehat{x})$ be the boundary defining function of domain $D^{i}, i=1,2$. Up to a translation in $(r, \widehat{x})$, there exists one pair of incident directions $\left\{\widehat{x}_{0},-\widehat{x}_{0}\right\}$ and $\left\{\widehat{x}_{0}^{\prime},-\widehat{x}_{0}^{\prime}\right\}$ in $\mathbb{S}^{2}$, respectively, such that $R^{1}( \pm \hat{x})=R^{2}( \pm \hat{x})$ in the neighborhood of $\widehat{x}_{0}$ and $\widehat{x}_{0}^{\prime}$, respectively.

Proof. By Theorem 6 assumption, we can assume the eigenvalues of $n^{1}$ and $n^{2}$ coincide for some $\pm \widehat{x}_{0}$ and $\pm \widehat{x}_{0}^{\prime}$, respectively, in $\mathbb{S}^{2}$. Considering some algebraic additions of (46) and (47), or (47) and (48), the boundary defining function at $\hat{x}$ appears as a part of eigenvalue asymptotics above. That is, $R^{1}( \pm \hat{x})=R^{2}( \pm \hat{x})$ for $\widehat{x}$ near $\hat{x}_{0}$ and $\hat{x}_{0}^{\prime}$, respectively. This proves the lemma.

Proposition 9. Let us assume $n^{1}(0)=n^{2}(0)=1$. There exists one fixed pair of incident directions $\left\{\widehat{x}_{0},-\widehat{x}_{0}\right\}$ and $\left\{\widehat{x}_{0}^{\prime},-\widehat{x}_{0}^{\prime}\right\}$ in $\mathbb{S}^{2}$, respectively, such that $n^{1}( \pm r \widehat{x}) \equiv n^{2}\left( \pm r \widehat{x}^{\prime}\right)$ for $\widehat{x}$ near $\widehat{x}_{0}$ and $\widehat{x}^{\prime}$ near $\hat{x}_{0}^{\prime}$, respectively.

Proof. Applying Lemmas 7 and 8, this is the previous uniqueness result from $[9-11,26]$. If two indices of refraction have an identical set of interior transmission eigenvalues along one of their incident directions, then they must be identical on that ray.

We deduce from the Weierstrass-Bolzano property on $\mathbb{S}^{2}$ that there are finitely many of such neighborhoods on $\mathbb{S}^{2}$ described in Lemmas 7 and 8.

Lemma 10. The density function $\Delta(\widehat{x})$ has the derivatives on $\partial D$ :

$$
\begin{aligned}
& \partial_{\theta} \Delta(\widehat{x})=\frac{2}{\pi} \partial_{\theta} R(\widehat{x}) ; \\
& \partial_{\varphi} \Delta(\widehat{x})=\frac{2}{\pi} \partial_{\varphi} R(\widehat{x}), \quad \widehat{x} \in \mathbb{S}^{2} .
\end{aligned}
$$

In particular, $\partial_{\theta} \Delta(\hat{x})$ and $\partial_{\varphi} \Delta(\widehat{x})$ are invariant to the rigid translation of $(n, D)$.

Proof. Applying the chain rule to $\widehat{x}$ in (56) with $-\widehat{x}$ fixed and setting $n^{\prime}(\widehat{x} R(\widehat{x}))=0$,

$$
\begin{aligned}
& \partial_{\theta} \Delta(\widehat{x})=\frac{1}{\pi} \partial_{\theta} R(\widehat{x})[1+\sqrt{n(R(\widehat{x}))}] \\
& \partial_{\varphi} \Delta(\widehat{x})=\frac{1}{\pi} \partial_{\varphi} R(\widehat{x})[1+\sqrt{n(R(\widehat{x}))}]
\end{aligned}
$$

Observing the boundary conditions of $(1), n(R(\widehat{x}))=1, \forall \widehat{x} \in$ $\mathbb{S}^{2}$. Thus, we deduce (58) and (59). We note that $\partial_{\theta} \Delta(\hat{x})$ and $\partial_{\varphi} \Delta(\hat{x})$ depend only on the boundary $\partial D$. This proves the lemma.

Proposition 11. Under the assumption of Theorem 6,

$$
\Delta^{1} \equiv \Delta^{2}, \quad \text { up to a translation }
$$


Proof. We deduce from Lemma 8, Proposition 9, and Lemma 10 that

$$
\begin{gathered}
\partial_{\theta} \Delta^{1}(\widehat{x})=\partial_{\theta} \Delta^{2}(\widehat{x}) \\
\partial_{\varphi} \Delta^{1}(\widehat{x})=\partial_{\varphi} \Delta^{2}(\widehat{x}) \\
R^{1}( \pm \hat{x})=R^{2}( \pm \widehat{x}) \\
n^{1}( \pm r \hat{x})=n^{2}( \pm r \widehat{x})
\end{gathered}
$$

near some $\widehat{x}_{0}$ and $\widehat{x}_{0}^{\prime}$, respectively, such that $R^{1}( \pm \widehat{x})=R^{2}( \pm \widehat{x})$ and $n^{1}(r \hat{x})=n^{2}(r \hat{x})$ up to a translation as well. For each index $n^{i}, i=1,2$, we collect all local coordinates such that (62) and (63) hold. As a result of the Weierstrass-Bolzano property on $\mathbb{S}^{2}$, there are mostly finitely many choices of neighborhoods of $\widehat{x}_{0}$ and $\hat{x}_{0}^{\prime}$ in which (62) and (63) hold, respectively, on $\partial D^{1}$ and $\partial D^{2}$, say, the collection of the neighborhoods

$$
\begin{aligned}
O^{1} & :=\left\{\xi_{1}, \xi_{2}, \ldots, \xi_{m}\right\} ; \\
O^{2} & :=\left\{\eta_{1}, \eta_{2}, \ldots, \eta_{n}\right\} .
\end{aligned}
$$

Because the boundary $D^{i}, i=1,2$, is given, all of the other neighborhoods satisfying (62) and (63) in each $\xi_{j}, j=$ $1, \ldots, m$, or $\eta_{j}, j=1, \ldots, n$ are known. Fix one of $\xi_{j_{0}}$ and $\eta_{j_{0}}$, the one is locally isomorphic to all other neighborhoods by applying (62), (63), (64), and (65) up to some translations. Accordingly, we choose a translation $\Psi: \mathbb{R}^{+} \times \mathbb{S}^{2} \rightarrow \mathbb{R}^{+} \times \mathbb{S}^{2}$ such that

$$
\Psi\left(R^{1}\right) \equiv R^{2}
$$

In this case, up to the chosen $\Psi$, we deduce that

$$
\begin{aligned}
& \partial_{\theta} \Delta^{1} \equiv \partial_{\theta} \Delta^{2} \\
& \partial_{\varphi} \Delta^{1} \equiv \partial_{\varphi} \Delta^{2}
\end{aligned}
$$

Applying (68) under Lemmas 10 and 7, we consider the initial value problems up to the translation $\Psi$ and deduce

$$
\begin{array}{cl}
\partial_{\theta} \Delta^{1}(\Psi(\widehat{x}))=\partial_{\theta} \Delta^{2}(\widehat{x}), & \widehat{x} \in \mathbb{S}^{2} ; \\
\partial_{\varphi} \Delta^{1}(\Psi(\widehat{x}))=\partial_{\varphi} \Delta^{2}(\widehat{x}), & \widehat{x} \in \mathbb{S}^{2} ; \\
\Delta^{1}\left(\Psi\left(\hat{x}_{0}\right)\right)=\Delta^{2}\left(\hat{x}_{0}^{\prime}\right) . &
\end{array}
$$

By the uniqueness of ODE of (58), (59), and (69), we obtain

$$
\Delta^{1} \equiv \Delta^{2}, \quad \text { up to the translation } \Psi \text {. }
$$

Surely we have $\partial D^{1} \equiv \partial D^{2}$ up to a translation by assumption, but Proposition 11 holds with some local information on the index of refraction.

Corollary 12. Let us assume $n^{i}$ and $R^{i}, i=, 1,2$, are analytical functions with the assumption of Theorem 6 without assuming that $\partial D^{1} \equiv \partial D^{2}$ up to a translation. If we assume the results of Lemmas 7 and 8, then we can conclude that

$$
\Delta^{1} \equiv \Delta^{2}, \quad \text { up to a translation. }
$$

Proof. If $n^{i}$ and $R^{i}, i=1,2$, are analytical functions, then (60) implies that $\Delta^{i}(\hat{x}), i=, 1,2$, are analytical in $\mathbb{S}^{2}$. Then (62) and (63) imply an analytic extension to $\mathbb{S}^{2}$. This and ODE (69) prove the corollary.

\section{Proof of Theorem 6 via the Doppler's Effect}

We want to show that, for a fixed $\widehat{x}$,

$$
\partial_{r} \Delta(\widehat{x})=\frac{1+\sqrt{n(r(\widehat{x}))}}{\pi}, \quad-R(-\widehat{x})<r<R(\widehat{x}) .
$$

We have the shifts of the frequency density to the space variable on the left hand side while the index of refraction is on the left hand side. Let us justify this differentiation by a perturbation theory on the index of refraction. We have seen the perturbation theory for finitely many interior transmission eigenvalues from $[7,9]$. As observed in $[9,10]$, the perturbation theory for finite eigenvalues implies a theory for all eigenvalues when considered with the asymptotic almost periodic structure (46), (47), and (48).

Let us consider a perturbation on the index of refraction in the form $n(r \widehat{x}) \chi_{[-R(-\widehat{x}), r]}$ where $\chi_{[-R(-\widehat{x}), r]}$ is the cutoff function defined on the interval $[-R(-\widehat{x}), r]$. We fix $-R(-\widehat{x})$ and start the perturbation at $r=R(\widehat{x})$. Outside $[-R(-\widehat{x}), r]$, we still take the index of refraction to be 1 . We can mollify the cusp of $n(r) \chi_{[-R(-\widehat{x}), r]}$ near $r$ in an arbitrarily small neighborhood by standard measure theory. Without loss of generality, we assume $n(r) \chi_{[-R(-\widehat{x}), r]}, i=1,2$, is differentiable, and the perturbation is carried out under the uniform norm. We recall from [9] the following theorem.

Theorem 13. Let $\left\{k_{j}\right\}$ be the zeros of $\widehat{D}_{0}(k)$. Then, for any $\sigma>$ 0 , there exists

$$
\eta=\sup \left|n^{\prime}-n\right|
$$

depending on $\sigma$, such that there exists an interior transmission eigenvalues of the index of refraction $n^{\prime}$, in $\sigma$-neighborhood of each $k_{j}$, whenever $\eta$ is small enough.

The perturbation theory for finitely many eigenvalues is firstly considered in [8]. Previously in $[9,10]$, we have shown from Sommerfeld's radiation condition (4), (5), and (26) that, along $\widehat{x}_{0}$ and $\widehat{x}_{0}^{\prime}$, respectively, for any common interior eigenvalue $k$ of index $n^{i}, i=1,2$, the following identities hold up to the translation $\Psi$ :

$$
\begin{gathered}
\widehat{y}_{l}^{1}\left(R^{1}\left(\widehat{x}_{0}\right) ; k\right)=\widehat{y}_{l}^{2}\left(R^{2}\left(\widehat{x}_{0}^{\prime}\right) ; k\right) ; \\
{\left[\widehat{y}_{l}^{1}\right]^{\prime}\left(R^{1}\left(\widehat{x}_{0}\right) ; k\right)=\left[\widehat{y}_{l}^{2}\right]^{\prime}\left(R^{2}\left(\widehat{x}_{0}^{\prime}\right) ; k\right) .}
\end{gathered}
$$

That is, the common interior transmission eigenvalues are the intersection points of two entire functions that move 
continuously to the perturbation to the index of refraction on either side of (74) according to Theorem 13.

Following [10, (2.36)] and [9], the zero density of entire functions

$$
\widehat{y}_{l}^{1}\left(R^{1}\left(\widehat{x}_{0}\right) ; k\right)-\widehat{y}_{l}^{2}\left(R^{2}\left(\widehat{x}_{0}^{\prime}\right) ; k\right)
$$

or

$$
\left[\widehat{y}_{l}^{1}\right]^{\prime}\left(R^{1}\left(\widehat{x}_{0}\right) ; k\right)-\left[\widehat{y}_{l}^{2}\right]^{\prime}\left(R^{2}\left(\widehat{x}_{0}^{\prime}\right) ; k\right)
$$

is

$$
\max \left\{\frac{\int_{0}^{R^{1}\left(\widehat{x}_{0}\right)} \sqrt{n^{1}\left(\widehat{x}_{0} \rho\right)} d \rho}{\pi}, \frac{\int_{0}^{R^{2}\left(\hat{x}_{0}^{\prime}\right)} \sqrt{n^{2}\left(\hat{x}_{0}^{\prime} \rho\right)} d \rho}{\pi}\right\},
$$

while the density for the common zero set is

$$
\Delta_{\widehat{D}_{0}^{i}}(-\epsilon, \epsilon)=\frac{R^{i}\left(\widehat{x}_{0}\right)+\int_{0}^{R^{i}\left(\widehat{x}_{0}\right)} \sqrt{n^{i}\left(\widehat{x}_{0} \rho\right)} d \rho}{\pi}, \quad \begin{aligned}
& \\
& \quad i=1,2,
\end{aligned}
$$

according to the assumption of Theorem 6 and (52). This contradicts with (77), and so Cartwright's theory $[9,10,18-$ $20,26]$ suggests that

$$
\begin{gathered}
\widehat{y}_{l}^{1}\left(R^{1}\left(\widehat{x}_{0}\right) ; k\right) \equiv \widehat{y}_{l}^{2}\left(R^{2}\left(\widehat{x}_{0}^{\prime}\right) ; k\right) ; \\
{\left[\hat{y}_{l}^{1}\right]^{\prime}\left(R^{1}\left(\widehat{x}_{0}\right) ; k\right) \equiv\left[\widehat{y}_{l}^{2}\right]^{\prime}\left(R^{2}\left(\widehat{x}_{0}^{\prime}\right) ; k\right) .}
\end{gathered}
$$

We refer the details of (58) up to (63) to [10] or [19, p. 251] for a Cartwright theory. In particular, $n^{1}$ and $n^{2}$ have identical sets of norming constants and Dirichlet/Neumann eigenvalues for the Sturm-Liouville problem (15). Hence, [29, Theorem 1.2] implies $n^{1}=n^{2}$ along $\widehat{x}_{0}$ and $\hat{x}_{0}^{\prime}$, respectively.

Let us consider the perturbation on both sides of (79) in the following form:

$$
\begin{aligned}
n^{i}(r \widehat{x}) \chi_{\left[R^{i}(-\widehat{x}), r\right]} \longmapsto n^{i}(r \widehat{x}) \chi_{\left[R^{i}(-\widehat{x}), r^{\prime}\right]} & \\
& \\
& r^{\prime}<r<R^{i}(\widehat{x}) .
\end{aligned}
$$

We denote the new solutions again by $\widehat{y}_{l}^{i}, i=1,2$. For each $r^{\prime}$, Theorem 13 and (52) imply that the new density function of the common set of interior transmission eigenvalues equals to

$$
\begin{gathered}
\frac{r^{\prime}\left(\widehat{x}_{0}\right)+\int_{0}^{r^{\prime}\left(\hat{x}_{0}\right)} \sqrt{n^{1}\left(\hat{x}_{0} \rho\right)} d \rho}{\pi} \\
\operatorname{or} \frac{r^{\prime}\left(\widehat{x}_{0}^{\prime}\right)+\int_{0}^{r^{\prime}\left(\hat{x}_{0}^{\prime}\right)} \sqrt{n^{2}\left(\hat{x}_{0}^{\prime} \rho\right)} d \rho}{\pi} .
\end{gathered}
$$

Without loss of generality, we take $\widehat{x}_{0}=\widehat{x}_{0}^{\prime}$ due to (67).

Whenever $r^{\prime}$ is close to $r$, this overdetermined density (81) again, by Cartwright's theory, implies the identities for the new solutions.

$$
\begin{gathered}
\widehat{y}_{l}^{1}\left(r^{\prime} ; k\right) \equiv \widehat{y}_{l}^{2}\left(r^{\prime} ; k\right) ; \\
{\left[\widehat{y}_{l}^{1}\right]^{\prime}\left(r^{\prime} ; k\right) \equiv\left[\widehat{y}_{l}^{2}\right]^{\prime}\left(r^{\prime} ; k\right) .}
\end{gathered}
$$

Moreover, we deduce from (82) and (83) again that the perturbed index of refraction $n^{i}\left(r \widehat{x}_{0}\right) \chi_{\left[-R^{i}\left(-\widehat{x}_{0}\right), r^{\prime}\right]}, i=1,2$, have an identical set of interior transmission eigenvalues $[9,10]$. From (81) and Theorem 13,

$$
\begin{aligned}
& \frac{r^{\prime}\left(\widehat{x}_{0}\right)+\int_{0}^{r^{\prime}\left(\widehat{x}_{0}\right)} \sqrt{n^{1}\left(\widehat{x}_{0} \rho\right)} d \rho}{\pi} \\
& =\frac{r^{\prime}\left(\widehat{x}_{0}\right)+\int_{0}^{r^{\prime}\left(\widehat{x}_{0}\right)} \sqrt{n^{2}\left(\hat{x}_{0} \rho\right)} d \rho}{\pi} .
\end{aligned}
$$

The perturbation applies in $-R^{i}\left(-\widehat{x}_{0}\right)<r^{\prime}<R^{i}\left(\widehat{x}_{0}\right), i=1,2$.

We repeat the same argument to the other incident directions $\widehat{x} \in \mathbb{S}^{2}$. Thus, we apply Proposition 11 to consider the limit quotient for all $\widehat{x} \in \mathbb{S}^{2}$ :

$$
\lim _{r^{\prime} \rightarrow r} \frac{r^{\prime}(\widehat{x})-r(\widehat{x})+\int_{r(\widehat{x})}^{r^{\prime}(\widehat{x})} \sqrt{n^{i}(\widehat{x} \rho)} d \rho}{\pi\left(r^{\prime}(\widehat{x})-r(\widehat{x})\right)}, \quad i=1,2 .
$$

Thus, we apply the Lebesgue Differentiation Theorem to deduce that

$$
\partial_{r} \Delta^{i}(\widehat{x})=\frac{1+\sqrt{n^{i}(r(\widehat{x}))}}{\pi}, \quad r>-R^{i}(\widehat{x}), \forall \widehat{x} \in \mathbb{S}^{2} .
$$

From Proposition 11, we conclude that

$$
n^{1} \equiv n^{2} \quad \text { up to the translation } \Psi \text {. }
$$

\section{Conflicts of Interest}

The author declares that there are no conflicts of interest regarding the publication of this manuscript.

\section{References}

[1] D. Colton and P. Monk, "The inverse scattering problem for time-harmonic acoustic waves in an inhomogeneous medium," The Quarterly Journal of Mechanics and Applied Mathematics, vol. 41, no. 1, pp. 97-125, 1988.

[2] A. Kirsch, "The denseness of the far field patterns for the transmission problem," IMA Journal of Applied Mathematics, vol. 37, no. 3, pp. 213-225, 1986.

[3] D. Colton, L. Päivärinta, and J. Sylvester, "The interior transmission problem," Inverse Problems and Imaging, vol. 1, no. 1, pp. 13-28, 2007.

[4] D. Colton and R. Kress, Inverse Acoustic and Electromagnetic Scattering Theory, vol. 93 of Applied Mathematical Sciences, Springer-Verlag, Berlin, Germany, 2nd edition, 2013.

[5] D. Colton, A. Kirsch, and L. Päivärinta, "Far-field patterns for acoustic waves in an inhomogeneous medium," SIAM Journal on Mathematical Analysis, vol. 20, no. 6, pp. 1472-1483, 1989.

[6] B. P. Rynne and B. D. Sleeman, "The interior transmission problem and inverse scattering from inhomogeneous media," SIAM Journal on Mathematical Analysis, vol. 22, no. 6, pp. 17551762, 1991.

[7] F. Cakoni, D. Colton, and H. Haddar, "The interior transmission eigenvalue problem for absorbing media," Inverse Problems, vol. 28, no. 4, Article ID 045005, 15 pages, 2012. 
[8] F. Cakoni, D. Colton, and D. Gintides, "The interior transmission eigenvalue problem," SIAM Journal on Mathematical Analysis, vol. 42, no. 6, pp. 2912-2921, 2010.

[9] L.-H. Chen, "A uniqueness theorem on the eigenvalues of spherically symmetric interior transmission problem in absorbing medium," Complex Variables and Elliptic Equations, vol. 60, no. 2, pp. 145-167, 2015.

[10] L.-H. Chen, "On the inverse spectral theory in a nonhomogeneous interior transmission problem," Complex Variables and Elliptic Equations, vol. 60, no. 5, pp. 707-731, 2015.

[11] L.-H. Chen, "Inverse uniqueness in interior transmission problem and its eigenvalue tunneling in simple domain," Advances in Mathematical Physics, vol. 2016, Article ID 2438253, 9 pages, 2016.

[12] A. Kirsch, "On the existence of transmission eigenvalues," Inverse Problems and Imaging, vol. 3, no. 2, pp. 155-172, 2009.

[13] E. Lakshtanov and B. Vainberg, "Bounds on positive interior transmission eigenvalues," Inverse Problems, vol. 28, no. 10, Article ID 105005, 105005, 13 pages, 2012.

[14] J. R. McLaughlin and P. L. Polyakov, "On the uniqueness of a spherically symmetric speed of sound from transmission eigenvalues," Journal of Differential Equations, vol. 107, no. 2, pp. 351-382, 1994.

[15] R. P. Boas, Entire Functions, Academic Press, New York, NY, USA, 1954.

[16] M. L. Cartwright, "On the Directions of Borel of Functions which are Regular and of finite Order in an Angle," Proceedings of the London Mathematical Society, vol. 38, pp. 503-541, 1933.

[17] M. L. Cartwright, Integral Functions, Cambridge University Press, Cambridge, UK, 1956.

[18] P. Koosis, The Logarithmic Integral I, Cambridge University Press, New York, NY, USA, 1997.

[19] B. J. Levin, Distribution of Zeros of Entire Functions, Translations of Mathematical Monographs, American Mathematical Society, Providence, RI, USA, 1972.

[20] B. Ya. Levin, Lectures on Entire Functions, vol. 150 of Translations of Mathematical Monographs, American Mathematical Society, Providence, RI, USA, 1996.

[21] J. Sun, "Estimation of transmission eigenvalues and the index of refraction from Cauchy data," Inverse Problems, vol. 27, no. 1, Article ID 015009, 015009, 11 pages, 2011.

[22] F. Zeng, T. Turner, and J. Sun, "Some results on electromagnetic transmission eigenvalues," Mathematical Methods in the Applied Sciences, vol. 38, no. 1, pp. 155-163, 2015.

[23] L. H. Chen, "A fixed energy fixed angle inverse scattering in interior transmission problem," Reports on Mathematical Physics, In press.

[24] T. Aktosun, D. Gintides, and V. G. Papanicolaou, "The uniqueness in the inverse problem for transmission eigenvalues for the spherically symmetric variable-speed wave equation," Inverse Problems, vol. 27, no. 11, Article ID 115004, 17 pages, 2011.

[25] T. Aktosun and V. G. Papanicolaou, "Reconstruction of the wave speed from transmission eigenvalues for the spherically symmetric variable-speed wave equation," Inverse Problems, vol. 29, no. 6, Article ID 065007, 19 pages, 2013.

[26] L.-H. Chen, "An uniqueness result with some density theorems with interior transmission eigenvalues," Applicable Analysis, vol. 94, no. 8, pp. 1527-1544, 2015.

[27] J. Pöschel and E. Trubowitz, Inverse Spectral Theory, Academic Press, Orlando, Fla, USA, 1987.
[28] R. Carlson, "Inverse spectral theory for some singular SturmLiouville problems," Journal of Differential Equations, vol. 106, no. 1, pp. 121-140, 1993.

[29] R. Carlson, "A Borg-Levinson theorem for BESsel operators," Pacific Journal of Mathematics, vol. 177, no. 1, pp. 1-26, 1997.

[30] V. A. Marchenko, Sturm-Liouville Operators and Applications, vol. 22, Birkhaeuser Verlag, Basel, Switzerland, 1986.

[31] http://mathworld.wolfram.com/Tangent.html.

[32] D. G. Dickson, "Zeros of exponential sums," Proceedings of the American Mathematical Society, vol. 16, pp. 84-89, 1965.

[33] D. G. Dickson, Expansions in Series of Solutions of Linear Difference-Differential and Infinite Order Differential Equations with Constant Coefficients, vol. 23, Memoirs of the American Mathematical Society, Providence, RI, USA, 1957. 


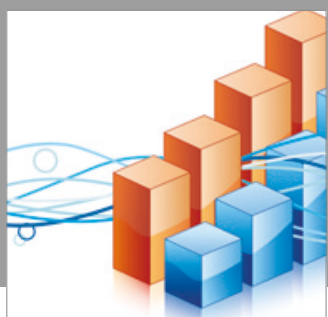

Advances in

Operations Research

vatersals

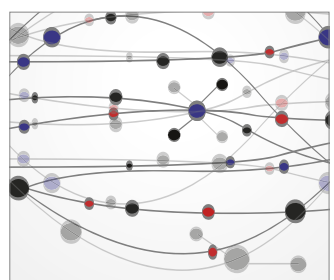

\section{The Scientific} World Journal
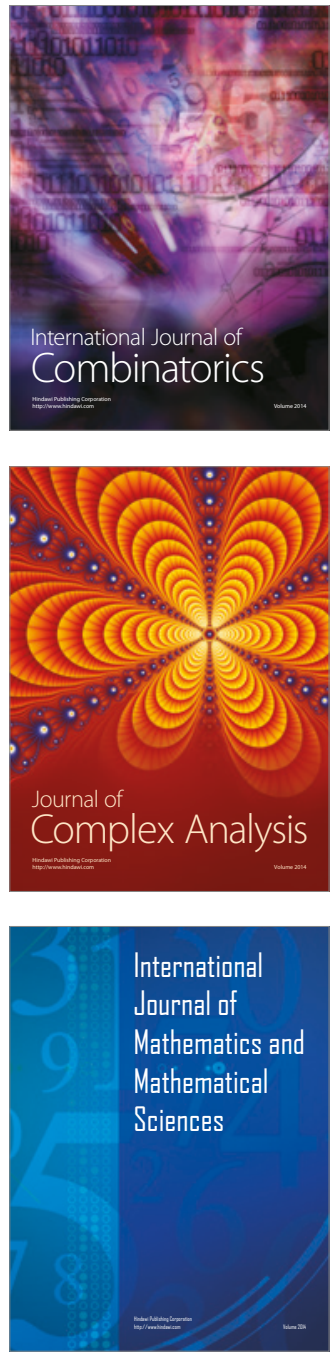
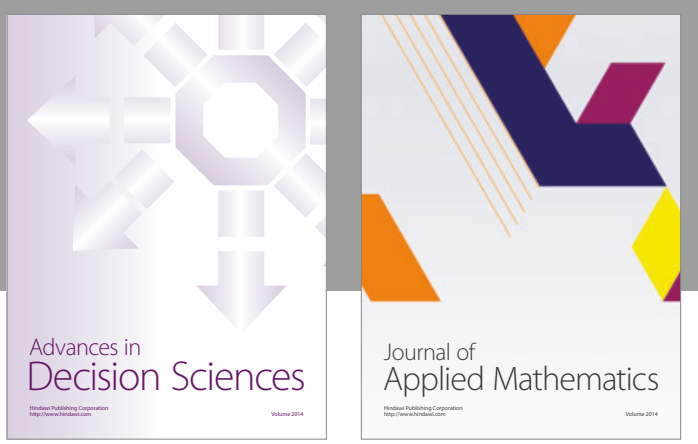

Algebra

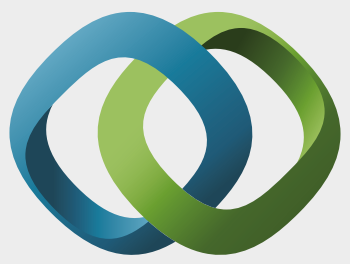

\section{Hindawi}

Submit your manuscripts at

https://www.hindawi.com
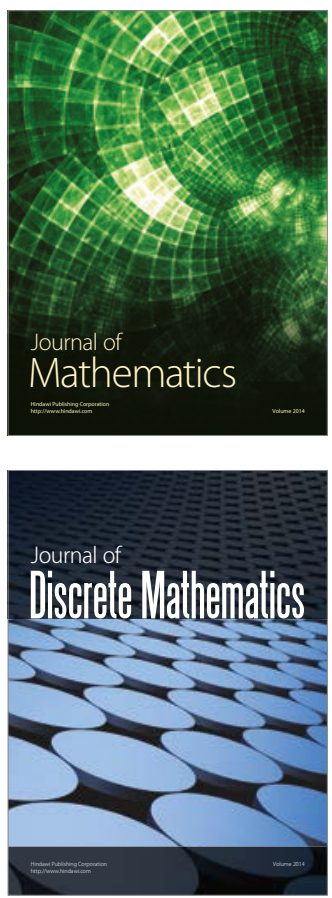

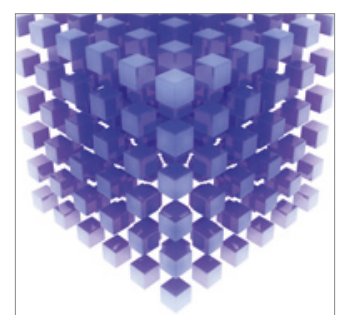

Mathematical Problems in Engineering
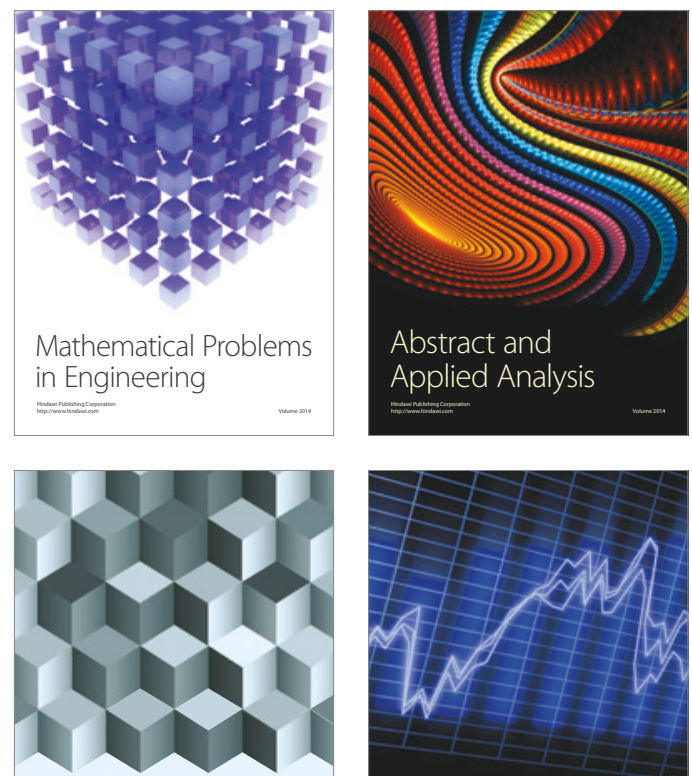

Journal of

Function Spaces

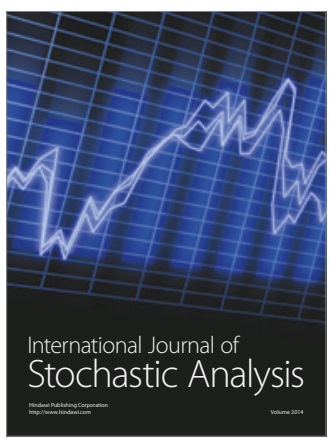

Probability and Statistics
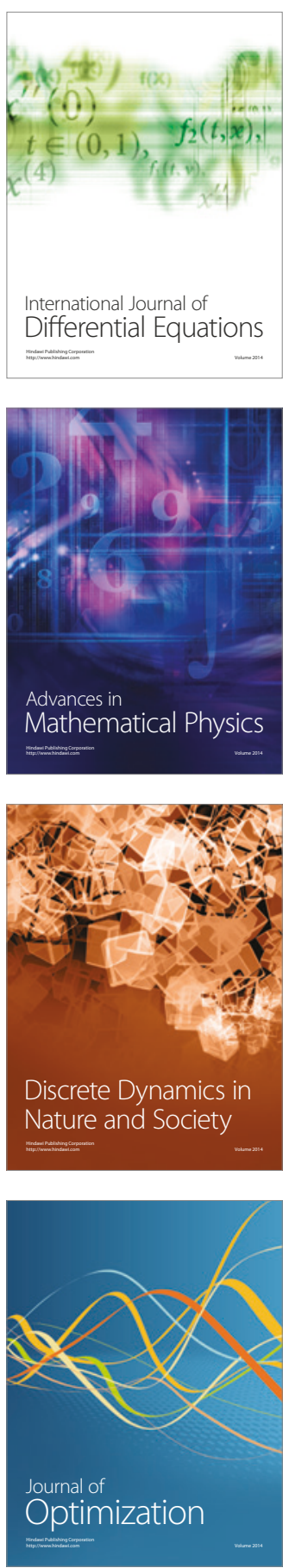